\title{
Editorial: Interplay Between RNA Processing Machinery and Epigenetic Regulation in Gene Expression
}

\author{
Naoyuki Kataoka ${ }^{1 *}$ and William C. Cho ${ }^{2}$ \\ ${ }^{1}$ Laboratory of Cellular Biochemistry, Department of Animal Resource Sciences, Graduate School of Agriculture and Life \\ Sciences, The University of Tokyo, Tokyo, Japan, ${ }^{2}$ Department of Clinical Oncology, Queen Elizabeth Hospital, Hong Kong, China
}

Keywords: RNA processing, mRNA, IncRNA, miRNA, epigenetic regulation

Editorial on the Research Topic

Interplay Between RNA Processing Machinery and Epigenetic Regulation in Gene Expression

In higher eukaryotes, gene expression consists of many steps such as transcription, mRNA processing, RNA transport and translation. Actually these steps are not fully independent, but they affect each other. It has been well accepted that there is the interplay between mRNA splicing and other gene expression steps (Boehm and Gehring, 2016; Schlautmann and Gehring, 2020).

There are accumulating lines of evidence that the epigenetic modifications in DNAs, histones and non-coding RNAs regulate not only RNA processing steps, but also other steps such as cell proliferation. Moreover, the defects in RNA processing and epigenetic regulation could result in many human diseases. Thus, this research topic aims to uncover novel interplay between epigenetics and RNAs in gene expression steps and diseases in human.

Pluripotent stem cells are good models for both application to regenerative medicine and to understand the biological process of embryonic development. Chen et al. focused on the role of lncRNA in stem cell fate through epigenetic regulation. Oct 4 and Sox 2 are well known transcription factors as members of Yamanaka-factors. They discussed the role of the Oct4 and Sox 2 promoter-interacting lncRNA identified by Chromatin RNA In Situ reverse Transcription sequencing (CRIST-seq). LncRNAs play important roles in stem cell pluripotency maintenance, differentiation and their dysfunction-related human diseases through different mechanisms. Recently, many lncRNAs were discovered to maintain stem cell pluripotency and determine their lineage differentiation. It was found that the sequence conservation, the RNA structure and the location of those lncRNAs are likely good indicators for their biological functions in cells and organs. Future studies to identify diseaseresponsible lncRNAs with information for their functions may identify good candidates for therapeutic approaches for some hereditary diseases.

LncRNAs also serve as "sponge" to absorb another functional noncoding RNA, miRNA. Wu et al. characterized the regulatory network among YAP1 and its targeted miRNAs and lncRNAs in $\mathrm{Hu}$ sheep SMSCs. YAP/TAZ act in the Hippo pathway, and YAP1 is able to regulate cell proliferation, migration, and apoptosis. While miR-29a significantly reduces the mRNA and protein expression level by binding to a specific $3^{\prime}$-UTR of YAP1, lncRNA CTTN-IT1 restores the expression of YAP1 through competitive binding to miR-29a. Furthermore, CTTN-IT1 can promote the proliferation and differentiation of SMSCs. A CTTN-IT1-miR-29a-YAP1 regulatory network contributes new insights into improving the muscle development of $\mathrm{Hu}$ sheep.

There is another "sponge" RNA produced by back-splicing, which is called as circular RNA (circRNA) (Sharma et al., 2021; Verduci et al., 2021). CircRNA plays an important role in tumorigenesis and progression of non-small cell lung cancer (NSCLC). Sun et al. investigated differentially expressed 
circRNAs and identified the pathogenesis hub genes of NSCLC by comprehensive bioinformatics analysis. They found 10 hub genes, at which the expression of MYLIP, GAN, and CDC27 were related to NSCLC patient prognosis. The relationship among circRNAsmiRNAs-target genes may provide novel insights for unraveling pathogenesis of NSCLC.

The other famous non-coding RNA, microRNA, which is much shorter than lncRNA and mRNA, also have critical roles in many biological steps, such as proliferation and development. Luo et al. described that miR-222 overexpression repressed cell cycle progression and proliferation, and it promoted the apoptosis of immature porcine Sertoli cells. miR-222 directly targeted the $3^{\prime} \mathrm{UTR}$ of the GRB10 mRNA and reduced its abundance. Their results indicated that miR-222 suppresses immature porcine Sertoli cell growth by targeting the GRB10 gene through inactivation of the PI3K/AKT signaling pathway. This study provided novel insights into the epigenetic regulation of porcine spermatogenesis by determining the fate of Sertoli cells.

Wang et al. investigated the regulatory mechanism of miR-143$3 p$ and lncRNA RP11-363N22.3-functioning upstream of KRAS-in exosomes derived from human mesenchymal stem cells (hMSCs) in pancreatic cancer. The exosomes are novel tools for cell-cell communications that contain RNAs and proteins. It is likely that miR-143-3p regulates KrasG12D, PI3K, ERK, JNK, p38MAPK, and vimentin synergistically to promote apoptosis and suppress cell growth, invasion, and migration in pancreatic cancer.

On the other hand, Wang et al. investigated Renal ischemia-reperfusion injury (IRI) and acute kidney injury (AKI). To determine the biological function and molecular mechanism of action of miR-92a-3p, they assessed the relationship between nuclear factor-erythroid 2-related factor 1 (Nrf1) and TEC pyroptosis induced by ischemia-reperfusion in vivo and oxygen-glucose deprivation/reoxygenation (OGD/ $\mathrm{R})$. They found that Nrf1 is a potential target for miRNA miR92a-3p, and the inhibition of miR-92a-3p alleviated oxidative stress in vitro and decreased the expression levels of NLRP3,

\section{REFERENCES}

Boehm, V., and Gehring, N. H. (2016). Exon Junction Complexes: Supervising the Gene Expression Assembly Line. Trends Genet. 32, 724-735. doi:10.1016/ j.tig.2016.09.003

Schlautmann, L. P., and Gehring, N. H. (2020). A Day in the Life of the Exon Junction Complex. Biomolecules 10, 866. doi:10.3390/ biom 10060866

Sharma, A. R., Bhattacharya, M., Bhakta, S., Saha, A., Lee, S.-S., and Chakraborty, C. (2021). Recent Research Progress on Circular RNAs: Biogenesis, Properties, Functions, and Therapeutic Potential. Mol. Ther. Nucleic Acids 25, 355-371. doi:10.1016/j.omtn.2021.05.022

Verduci, L., Tarcitano, E., Strano, S., Yarden, Y., and Blandino, G. (2021). CircRNAs: Role in Human Diseases and Potential Use as Biomarkers. Cell Death Dis. 12, 468. doi:10.1038/s41419-021-03743-3 caspase-1, GSDMD-N, IL-1 $\beta$, and IL-18 in vitro and in vivo. The results suggested that the inhibition of miR-92a-3p lessen tubular epithelial cell oxidative stress and pyroptosis by targeting Nrfl in renal IRI.

Lastly, RNA processing is also coordinated with $3^{\prime}$ end processing. The mRNA type RNA including lncRNA undergoes $3^{\prime}$ end processing which is coupled with transcription termination in higher eukaryotes. The majority of eukaryotic genes are subject to alternative polyadenylation (APA) by utilizing multiple poly(A) sites. Soles and Shi summarized APA mechanism with cis-regulatory elements and trans-acting factors. In addition, they described important functions for epigenetic mechanisms in APA, including modifications of DNA and histones and higher-order chromatin structures. Although many of epigenetic regulators were shown to play important roles in APA regulation, it is still unclear how they affect RNA polymerase II processivity. Future studies on APA and epigenetics will contribute to identify "adaptors" that connect histones with APA factors to regulate polyadenylation. This will also provide information for the biological consequences of epigenetics-mediated APA regulation in development and in human diseases.

In summary, our research topic focuses on the interplay between biological steps and RNA network presents many interesting associations between epigenetics and RNAs, including miRNA, ceRNA, IncRNA and mRNA. RNA processing steps are not fully independent but linked with other gene expression steps in higher eukaryotes. Future studies to uncover more interplays will contribute to elucidate whole gene expression networks in eukaryotes.

\section{AUTHOR CONTRIBUTIONS}

All authors listed have made a substantial, direct, and intellectual contribution to the work and approved it for publication.

Conflict of Interest: The authors declare that the research was conducted in the absence of any commercial or financial relationships that could be construed as a potential conflict of interest.

Publisher's Note: All claims expressed in this article are solely those of the authors and do not necessarily represent those of their affiliated organizations, or those of the publisher, the editors and the reviewers. Any product that may be evaluated in this article, or claim that may be made by its manufacturer, is not guaranteed or endorsed by the publisher.

Copyright (c) 2021 Kataoka and Cho. This is an open-access article distributed under the terms of the Creative Commons Attribution License (CC BY). The use, distribution or reproduction in other forums is permitted, provided the original author(s) and the copyright owner(s) are credited and that the original publication in this journal is cited, in accordance with accepted academic practice. No use, distribution or reproduction is permitted which does not comply with these terms. 